\title{
ARTICLE
}

\section{Mice with a heterozygous Lrp6 deletion have impaired fracture healing}

\author{
Travis A Burgers ${ }^{1}$, Juan F Vivanco ${ }^{2}$, Juraj Zahatnansky ${ }^{1}$, Andrew J Vander Moren ${ }^{3}$, James J Mason ${ }^{1}$ and \\ Bart O Williams ${ }^{1}$
}

\begin{abstract}
Bone fracture non-unions, the failure of a fracture to heal, occur in $10 \%-20 \%$ of fractures and are a costly and debilitating clinical problem. The Wnt/ $\beta$-catenin pathway is critical in bone development and fracture healing. Polymorphisms of linking low-density lipoprotein receptor-related protein 6 (LRP6), a Wnt-binding receptor, have been associated with decreased bone mineral density and fragility fractures, although this remains controversial. Mice with a homozygous deletion of $\operatorname{Lrp} 6$ have severe skeletal abnormalities and are not viable, whereas mice with a heterozygous deletion have a combinatory effect with Lrp5 to decrease bone mineral density. As fracture healing closely models embryonic skeletal development, we investigated the process of fracture healing in mice heterozygous for $\operatorname{Lrp} 6\left(\operatorname{Lrp6}^{+/-}\right)$and hypothesized that the heterozygous deletion of $\operatorname{Lrp} 6$ would impair fracture healing. Mid-diaphyseal femur fractures were induced in $\operatorname{Lrp6^{+/-}}$ mice and wild-type controls $\left(\operatorname{Lrp6^{+/+}}\right)$. Fractures were analyzed using micro-computed tomography $(\mu \mathrm{CT})$ scans, biomechanical testing, and histological analysis. $\operatorname{Lrp6^{+/-}}$ mice had significantly decreased stiffness and strength at 28 days post fracture (PF) and significantly decreased BV/TV, total density, immature bone density, and mature area within the callus on day-14 and -21 PF; they had significantly increased empty callus area at days 14 and 21 PF. Our results demonstrate that the heterozygous deletion of Lrp6 impairs fracture healing, which suggests that Lrp6 has a role in fracture healing.
\end{abstract}

Bone Research (2016) 4, 16025; doi:10.1038/boneres.2016.25; published online: 6 September 2016

\section{INTRODUCTION}

The $W n t / \beta$-catenin pathway is critical in bone development. ${ }^{1-2}$ The importance of this pathway to bone development was first demonstrated with a report linking lowdensity lipoprotein receptor-related protein 5 (LRP5) mutations and the pediatric syndrome, osteoporosis pseudoglioma. ${ }^{3}$ Soon after, two studies showed that point mutations in LRP5 cause increased bone mass. ${ }^{4-5}$ The $\mathrm{Wnt} / \beta$-catenin pathway is initiated when a Wnt protein that binds to the complex that includes Lrp5, Lrp6, and Frizzled. ${ }^{6}$ Downstream, this allows $\beta$-catenin to avoid ubiquitindependent destruction that occurs through disheveled and glycogen synthase kinase 3 . Increased levels of $\beta$-catenin cause nuclear translocation that causes TCF (transcription factor)/LEF (lymphoid enhancer-binding factor)- and TAZ (transcriptional coactivator with a PDZbinding domain)-mediated gene transcription. ${ }^{7-8}$

Polymorphisms of LRP6 have been associated with decreased bone mineral density and fragility fractures, ${ }^{9-12}$ although others have not confirmed the results. ${ }^{13-15}$ Mice with a homozygous deletion of Lrp6 have severe skeletal abnormalities and are not viable. ${ }^{16}$ Mice with heterozygous Lrp6 deletion have a combinatory effect with Lrp5 to decrease bone mineral density. ${ }^{17-18}$

Similar to embryonic skeletal development, ${ }^{19}$ fracture repair utilizes regulated chondrogenic and osteoblastic phases of bone formation: first, inflammation; second, cartilaginous callus formation; third, endochondral ossification; and fourth, bone remodeling. ${ }^{20} \mathrm{~A}$ non-union occurs if this process is disturbed and the fracture healing lasts

\footnotetext{
${ }^{1}$ Center for Cancer and Cell Biology, Program for Skeletal Disease and Tumor Microenvironment, Van Andel Research Institute, Grand Rapids, MI, USA; ${ }^{2}$ Facultad de Ingenieria y Ciencias, Adolfo Ibáñez University, Viña del Mar, Chile and ${ }^{3}$ Padnos College of Engineering and Computing, Grand Valley State University, Grand Rapids, MI, USA
}

Correspondence: Bart O Williams (bart.williams@vai.org)

Received: 25 April 2016; Revised: 17 May 2016; Accepted: 22 June 2016 
longer or does not complete at all. Non-unions occur in $10 \%-20 \%$ of fractures ${ }^{21-22}$ and lead to increased treatment costs and patient morbidity. ${ }^{23}$ The $\mathrm{Wnt} / \beta$-catenin pathway is also important in fracture healing. ${ }^{20,24-26}$ Mice with a homozygous deletion of Lrp5 have impaired fracture healing, ${ }^{27}$ whereas mice with a homozygous deletion of secreted frizzled-related protein 1, a Wnt/ $\beta$-catenin inhibitor, have improved fracture healing. ${ }^{28}$ To the authors' knowledge, there have been no studies investigating the role of Lrp6 in fracture healing.

Owing to the importance of the Wnt/ $\beta$-catenin signaling pathway, specifically LRP6, on bone development and the importance of the $W n t / \beta$-catenin signaling pathway in fracture healing, we hypothesize that the heterozygous deletion of Lrp6 will impair fracture healing.

\section{MATERIALS AND METHODS}

Animal model and specimen preparation

This study was approved by the Institutional Animal Care and Use Committee at the Van Andel Research Institute (Grand Rapids, MI, USA). Mice on a C57BL/6J background with a heterozygous deletion of $\operatorname{Lrp6}\left(\mathrm{Lrp6}^{+/-}\right)$and wildtype control $\left(\mathrm{Lrpb}^{+/+}\right)$mice were previously generated. ${ }^{29}$ To examine fracture healing and biomechanical characteristics, surgery was performed on approximately equivalent numbers of male and female mice that were 11-12 weeks old, and the right femur was fractured.

Each mouse was anesthetized using a subcutaneous weight-matched dose of tribromoethanol (average $350 \mu \mathrm{L}$ dose of $0.079 \mathrm{mg} \cdot \mu \mathrm{L}^{-1}$ solution; Avertin; Winthrop Laboratories, New York, NY, USA). A 23-gauge needle was surgically inserted in the femoral medullary canal of the right femur and a femoral fracture was created at the midshaft using a blunt impact force in a three-point bending technique, following an established procedure. ${ }^{30}$ Pain was managed postoperatively with subcutaneous doses of tramadol ${ }^{31}\left(20 \mathrm{mg} \cdot \mathrm{kg}^{-1}\right.$; Sigma-Aldrich, St Louis, MO, USA) administered at the time of surgery and at 12, 24, and $36 \mathrm{~h}$ after fracture. Mice were killed at 7, 14, 21, and 28 days post fracture (PF). Mice were divided into one group for biomechanical and micro-computed tomography $(\mu \mathrm{CT})$ evaluation $(n=99)$ and one group for histological evaluation $(n=45)$. For the biomechanical and $\mu \mathrm{CT}$ evaluation, 34 fractured femurs (from $16 \mathrm{Lrpb}^{+/+}$and $18 \mathrm{Lrp6}^{+/-}$ animals) were excluded because the fracture was oblique, comminuted, or incomplete, as determined by follow-up radiographs. $^{32}$

Biomechanical evaluation

After killing of each animal for biomechanical and $\mu \mathrm{CT}$ analyses, both the fractured and intact femurs were excised and cleaned of the surrounding soft tissue. The intramedullary needle in the fractured femur was removed and samples were stored at $-20^{\circ} \mathrm{C}$ in salinesaturated gauze.

For biomechanical assessment of the bones, all femurs were removed from the freezer, rehydrated in saline, and allowed to equilibrate to room temperature. Four-point bending mechanical testing was performed on the right femur according to the same procedure as our previous work. ${ }^{32}$ Force and displacement were directly measured using the TestResources (TestResources, Shakopee, MN, USA) system. Stiffness (defined as the ratio between force and displacement in the representative linear region) and maximum strength (maximum load) were calculated in Excel (Microsoft, Redmond, WA, USA). The coefficient of determination $\left(R^{2}\right)$ of the stiffness measure was $0.9989 \pm 0.0015$ (average \pm s.d.). The stiffness ratio and maximum load ratio were determined as the ratio of the respective fractured femur property to the intact femur property.

$\mu \mathrm{CT}$ evaluation

For $\mu \mathrm{CT}$ analysis, all femurs were scanned in saline by $\mu \mathrm{CT}$ using a Skyscan 1172 high-resolution micro-CT (Skyscan, Kontich, Belgium) with a voxel size of $13.3 \mu \mathrm{m}$. The two Skyscan calibration phantoms were included in each scan. Images were reconstructed using the Skyscan software. Mimics 14.11 (Materialise, Ann Arbor, MI, USA) was used to segment the phantoms and fracture calluses. ${ }^{32}$ The linear relationship between the bone mineral content and Hounsfield units $(\mathrm{HU})^{33-34}$ of each scan was calculated using the known density of the two calibration phantoms $\left(0.25\right.$ and $0.75 \mathrm{~g} \cdot \mathrm{cm}^{-3}$ ) and their segmented $\mathrm{HU}$ values.

In Mimics, two transverse slices were used to analyze the bone maturity level of each callus (see Burgers et al. ${ }^{32}$ and Collins et al. ${ }^{35}$ for similar examples). The center of the callus was determined as the location of the small gap between the bones. One slice each was used $0.5 \mathrm{~mm}$ proximal and distal to the determined center. ${ }^{32}$ For each slice, four masks were created. The first mask included the total callus crosssectional area by differentiating the boundary of the callus from the saline using the Mimics thresholding and "3D LiveWire" tools. The second included only the mature area, described by Komatsu et al. ${ }^{27}$ as that with a density greater than $600 \mathrm{mg} \cdot \mathrm{cm}^{-3}$. The third included only the immature bone area, described by Komatsu et al. ${ }^{27}$ as that with a density of $250-600 \mathrm{mg} \cdot \mathrm{cm}^{-3}$. The fourth was the empty (not mineralized) area with a density less than $250 \mathrm{mg} \cdot \mathrm{cm}^{-3}$. The second through fourth masks were defined using a Boolean operation with the applicable thresholded density range. The total, mature and immature densities of each region were determined by calculating the mineral content divided by the volume (area times slice thickness) of 
each mask. Mature bone volume over tissue volume (BV/TV) was calculated as the ratio of the mature area to the total area of the callus. Immature bone volume over tissue volume (IV/TV) was calculated as the ratio of the immature area to the total area of the callus.

Histological evaluation

For histological analysis, femurs were excised, the needle was removed, and the sample was fixed in $10 \%$ neutral buffered formalin for at least $24 \mathrm{~h}$, and decalcified in $10 \%$ EDTA, pH 7.5 for 5-8 days at room temperature. The samples were embedded in paraffin along their long axis and sectioned $(5 \mu \mathrm{m})$. Before staining, slides were deparaffinized and gradually rehydrated though series of ethanol washes. Sections were stained for immunohistochemistry $(\mathrm{IHC})$ and tartrate-resistant acid phosphatase (TRAP). For IHC, in the antigen-retrieval step, slides were incubated in pre-boiled citrate buffer (\#005001, Life Technologies, Grand Island, NY, USA) for $10 \mathrm{~min}$. Endogenous peroxidase activity was quenched by $3 \%$ hydrogen peroxide for $30 \mathrm{~min}$. The slides were then incubated overnight with primary rabbit anti- $\beta$ catenin antibody (\#9562, Cell Signaling, Danvers, MA, USA) diluted 1:200. The secondary biotinylated goat anti-rabbit antibody (\#BA-1000, Vector Laboratories, Burlingame, CA, USA) diluted 1:200 was used with the Vectastain avidin biotinylated enzyme complex system for visualization. TRAP staining for osteoclasts was performed using a Leukocyte Acid Phosphatase Kit (\#387A, Sigma-Aldrich) and counterstained with hematoxylin.

Statistical analyses

The effect of genotype was assessed statistically. Twotailed Student's t-tests were performed in Excel assuming unequal variance between groups, and $P<0.05$ was considered significant. Average and s.d. are reported.

\section{RESULTS}

The stiffness and maximum strength (maximum load) were measured in $\mathrm{Lrp6}^{+/-}$and $\mathrm{Lrp6}^{+/+}$femurs intact (Figure 1) and during the process of fracture repair (Figure 2). There was no significant difference in the intact contralateral bones from the $\mathrm{Lrp6}^{+/-}$and $\mathrm{Lrp6}^{+/+}$controls at any time point.

As expected, the stiffness and strength increased throughout healing in both $\mathrm{Lrpb}^{+/-}$and $\mathrm{Lrpb}^{+/+}$groups (Figure 2). Relative to $\mathrm{Lrp6}^{+/+}$mice, $\mathrm{Lrp6}^{+/-}$mice had significantly lower stiffness and maximum strength at 28 days PF. The stiffness and maximum load in the $\mathrm{Lrpb}^{+/-}$group were decreased to 68 and $80 \%$ of the $\mathrm{Lrpb}^{+/+}$group, respectively, at day 28 PF.

At days 14 and $21 \mathrm{PF}$, the ratio of the biomechanical properties (stiffness and maximum load) of the fractured to intact limb was not significantly different between the $\mathrm{Lrp6}^{+/-}$and $\mathrm{Lrp6}^{+/+}$groups (Figure 3). At day $28 \mathrm{PF}$, the stiffness ratio was not significant between groups, but the $\mathrm{Lrp6}^{+/-}$had a significantly lower maximum load ratio $(75 \%$ of the $\mathrm{Lrpb}^{+/+}$group).

To gain further insight into how the heterozygous deletion of Lrp6 inhibits the late decrease in biomechanical characteristics of the healing bone, the $\mu \mathrm{CT}$ scans of the calluses during healing were examined. At days 14 and 21 $\mathrm{PF}$, the $\mathrm{Lrp6}^{+/-}$group had significantly less callus BV/TV $153 \%$ and $74 \%$ that of the $\mathrm{Lrp6}^{+/+}$group at days 14 and $21 \mathrm{PF}$, respectively) and IV/TV (72\% and $82 \%$, respectively; Figure 4). The $\mathrm{Lrp6}^{+/-}$group had significantly less total callus density ( $75 \%$ and $83 \%$, respectively) at days 14 and $21 \mathrm{PF}$ and less immature density at day $14 \mathrm{PF}(93 \%)$;
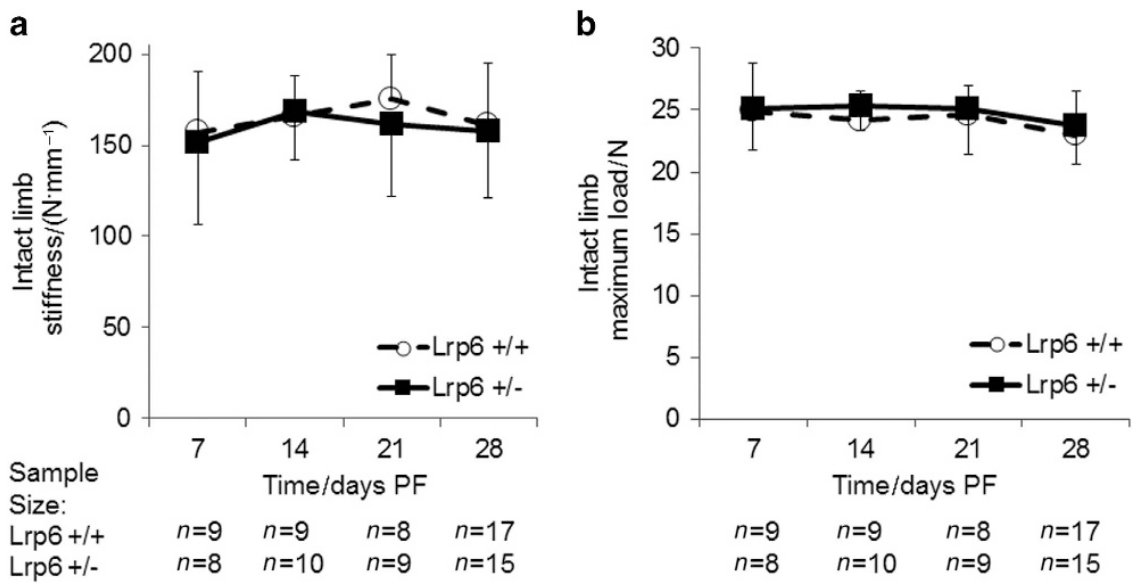

Figure 1. Biomechanical measures of intact bone. There was no statistical difference in (a) stiffness and (b) maximum load in $L r p 6^{+/-}$and $L r p 6^{+/+}$ controls at any time point. Error bars indicate one s.d. above the $\operatorname{Lrp} 6^{+/+}$group and one s.d. below the Lrp $6^{+/-}$group. 
a

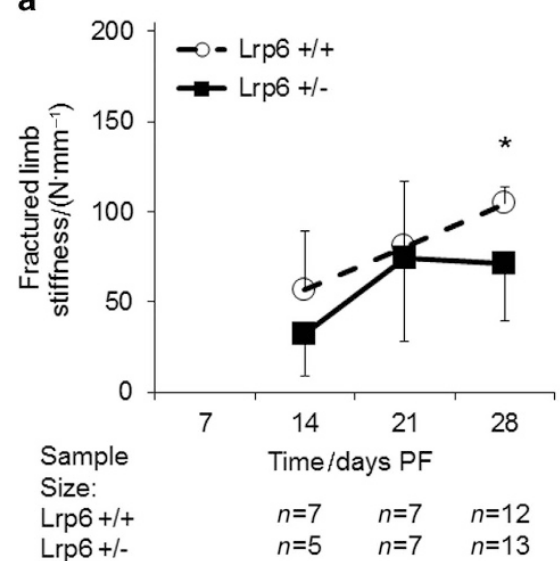

b

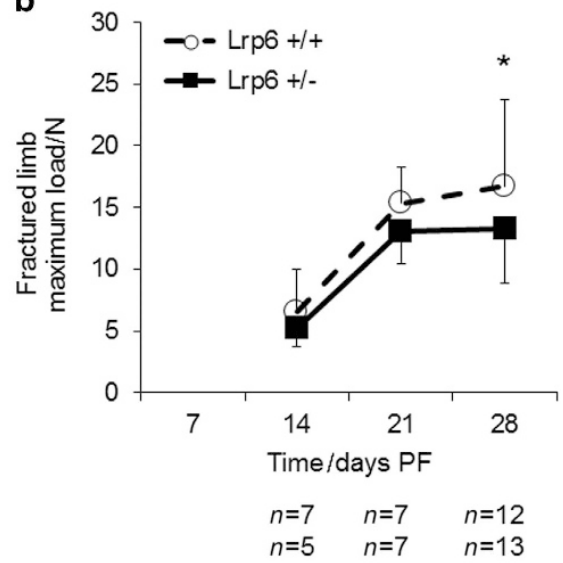

Figure 2. Biomechanical measures of fractured femurs. There was a significant decrease in the (a) stiffness and (b) maximum load at 28 days post fracture (PF) in the $\mathrm{Lrp}^{+/-}$group. Error bars indicate one s.d. above the $\mathrm{Lrp}^{+/+}$group and one s.d. below the $\operatorname{Lrp} 6^{+/-}$group ${ }^{*} \mathrm{P}<0.05, \mathrm{Lrp} 6^{+/-}$ and $\operatorname{Lrp}^{+/+}$at the time point).
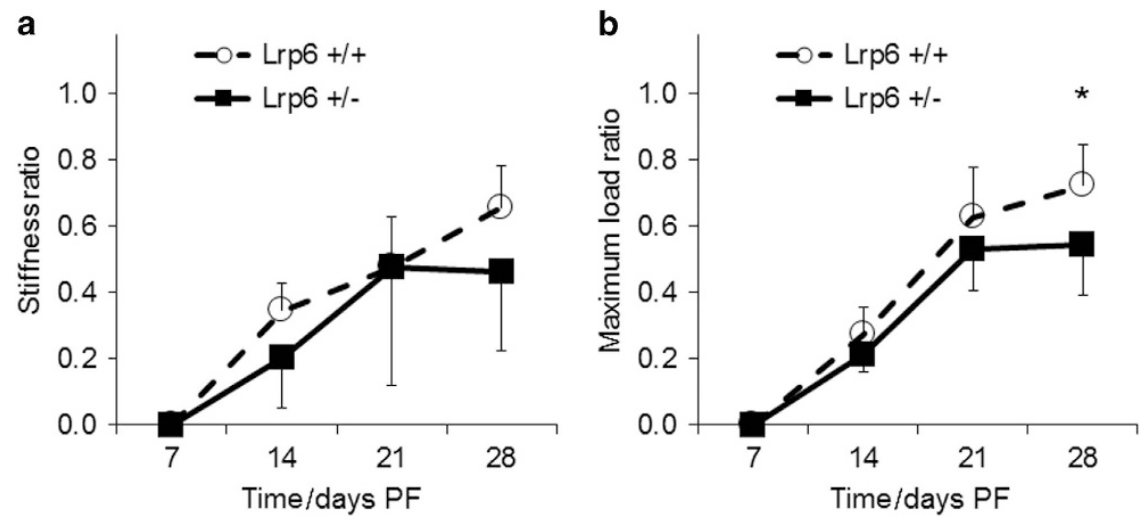

Figure 3. Stiffness and maximum load ratios of fractured to intact femurs. There was no statistical difference in the (a) stiffness load ratio at any time point. There was a significant decrease in the (b) maximum load ratio at 28 days post fracture (PF) in the $\operatorname{Lrp} 6^{+/-}$group. Error bars indicate one s.d. above the $\operatorname{Lrp}^{+/+}$group and one s.d. below the $\operatorname{Lrp}^{+/-}$group $\left({ }^{*} P<0.05, \operatorname{Lrp} 6^{+/-}\right.$and $\operatorname{Lrp} 6^{+/+}$at the time point).

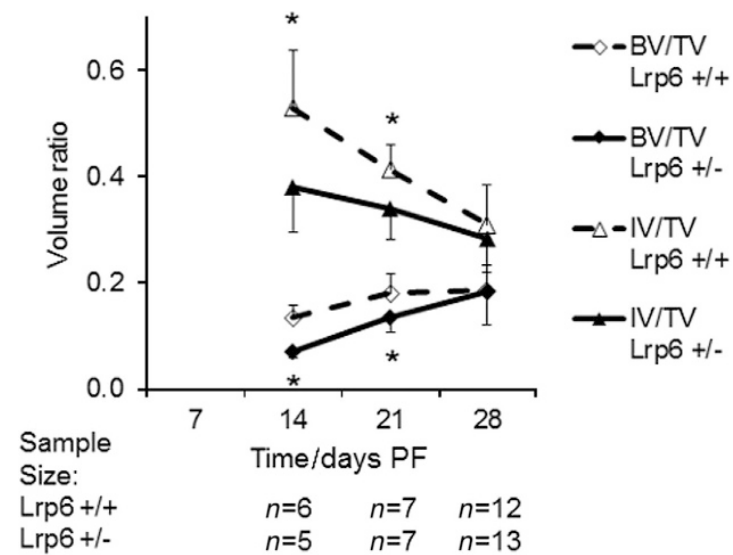

Figure 4. Bone volume over tissue volume (BV/TV) and immature bone volume over tissue volume (IV/TV) ratio for fracture callus. There was a significant decrease in BV/TV and IV/TV at days 14 and 21 post fracture (PF) in the $\mathrm{Lrp6}^{+/-}$group. Error bars indicate one s.d. above the $\mathrm{Lrp6}^{+/+}$group and one s.d. below the $\operatorname{Lrp} 6^{+/-}$group $\left({ }^{*} \mathrm{P}<0.05, \mathrm{Lrp6}^{+/-}\right.$ and $\mathrm{LrpG}^{+/+}$at the time point, and note that IV/TV significance is indicated below the data points). however, there was no significant difference in mature density at any time point (Figure 5). The $\mathrm{Lrp6}^{+/-}$group had significantly less mature area $(65 \%$ and $82 \%$, respectively) at days 14 and 21 PF and significantly more empty area $(198 \%$ and $139 \%$, respectively) at days 14 and 21 PF (Figure 6). There was no significant difference in total callus area or immature area at any time point (Figure 6).

To gain further insight into the biological differences within the fracture calluses due to the heterozygous deletion of Lrp6, IHC was performed. IHC sections showed no apparent qualitative differences stained for Wnt signaling activity ( $\beta$ catenin; Figure 7) or osteoclasts (TRAP; Figure 8) at any of the three time points (day 21 PF not shown).

\section{DISCUSSION}

Relative to the $\mathrm{Lrp6}^{+/+}$group, the $\mathrm{Lrpb}^{+/}$group had no significant difference in the stiffness and strength of intact 
a

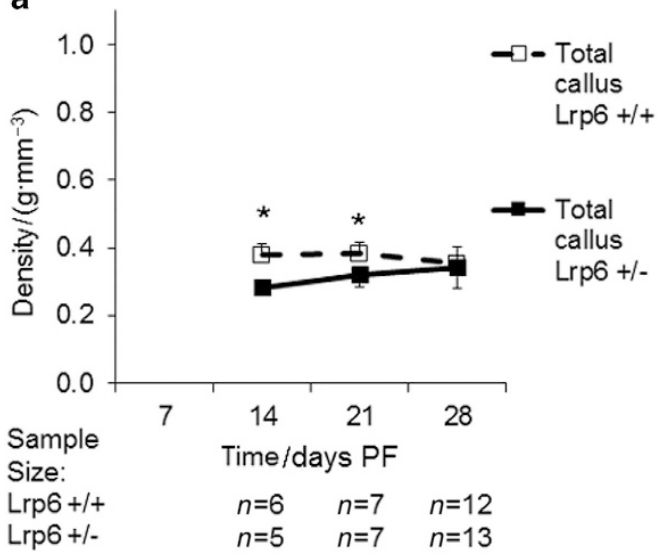

b

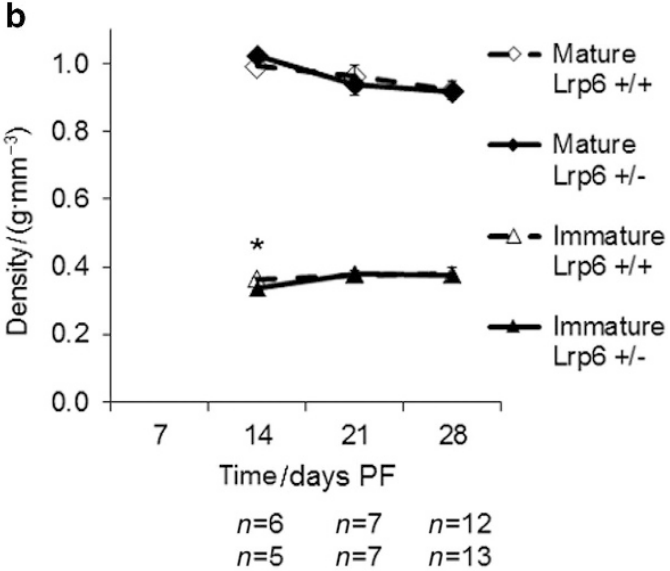

Figure 5. Total callus density, mature callus density, and immature callus density in the fracture callus. There was a significant decrease in (a) total callus density at days 14 and 21 post fracture (PF) in the $\mathrm{Lrp6}^{+/-}$group and in the (b) immature callus density at day $14 \mathrm{PF}$. There was no significant difference in the mature callus density at any time point. Error bars indicate one s.d. above the $L r p 6^{+/+}$group and one s.d. below the Lrp $6^{+/-}$group $\left({ }^{*} \mathrm{P}<0.05, \mathrm{Lrp6}^{+/-}\right.$and $\mathrm{Lrp6}^{+/+}$at the time point).

a

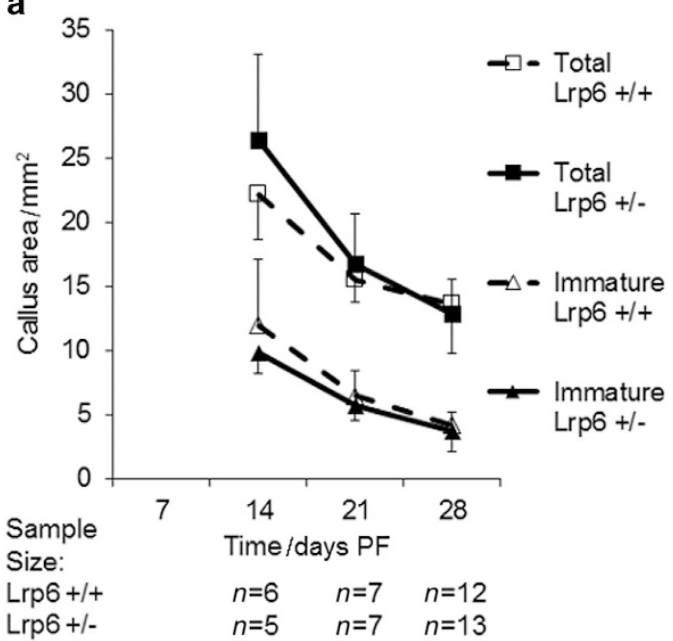

b

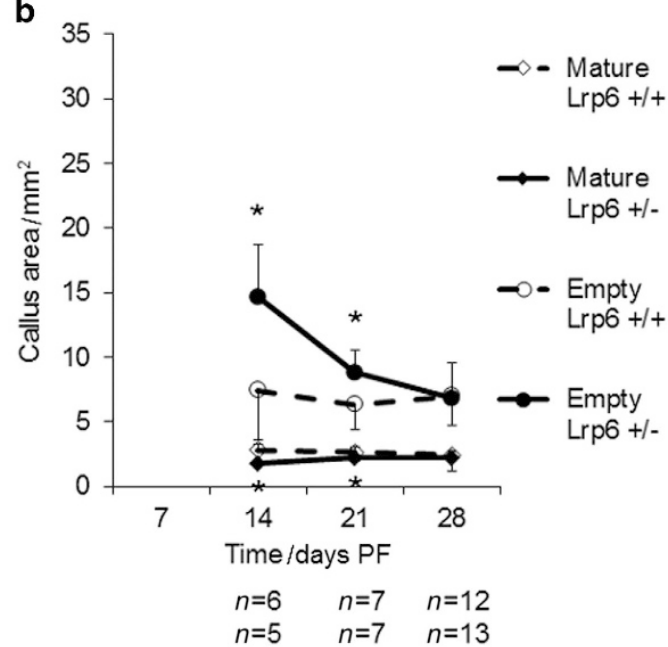

Figure 6. Total callus area, mature callus area, immature callus area, and empty callus area in the fracture callus. There was no statistical difference in the (a) total area or immature area at any time point. There was a significant increase in the (b) empty area and a significant decrease in the (b) mature area at days 14 and 21 post fracture (PF) in the $\mathrm{Lrp}^{+/-}$group. There was no statistical difference in total area or immature area at any time point. Error bars indicate one s.d. above for the group with the larger area and one s.d. below for the group with the smaller area $\left({ }^{*} P<0.05, \operatorname{Lrp} 6^{+/-}\right.$ and $\operatorname{Lrp6}^{+/+}$at the time point).

bones at all time points. In a previous study investigating limb development, the $\mathrm{Lrpb}^{+/-}$group had significantly decreased trabecular BV/TV compared with the $\mathrm{Lrpb}^{+/+}$ group. Other bone characteristics were decreased but not significantly so. ${ }^{17}$ The mechanical characteristics are consistent with this result.

Biomechanical analysis also showed that $\mathrm{Lrpb}^{+/-}$mice had significantly reduced stiffness and maximum strength at day 28 PF in the fractured limb. They also had a significantly reduced maximum load ratio of the fractured to intact limb at that time point. This difference later in the healing process demonstrates that the heterozygous deletion of Lrp6 disrupts at least one stage of endochondral ossification. Fracture healing was also disrupted because of the homozygous deletion of Lrp $5^{27}$ and the osteoblastspecific deletion of $\beta$-catenin. ${ }^{26}$ The results reported here along with the previous literature demonstrate the importance of the role of Wnt signaling in the callus osteoblasts and/or chondrocytes during fracture healing.

The $\mu \mathrm{CT}$ analysis showed that at days 14 and $21 \mathrm{PF}$, the time in which chondrogenesis and endochondral ossification is occurring, ${ }^{20}$ the $\mathrm{Lrpb}^{+/-}$group had significantly less 

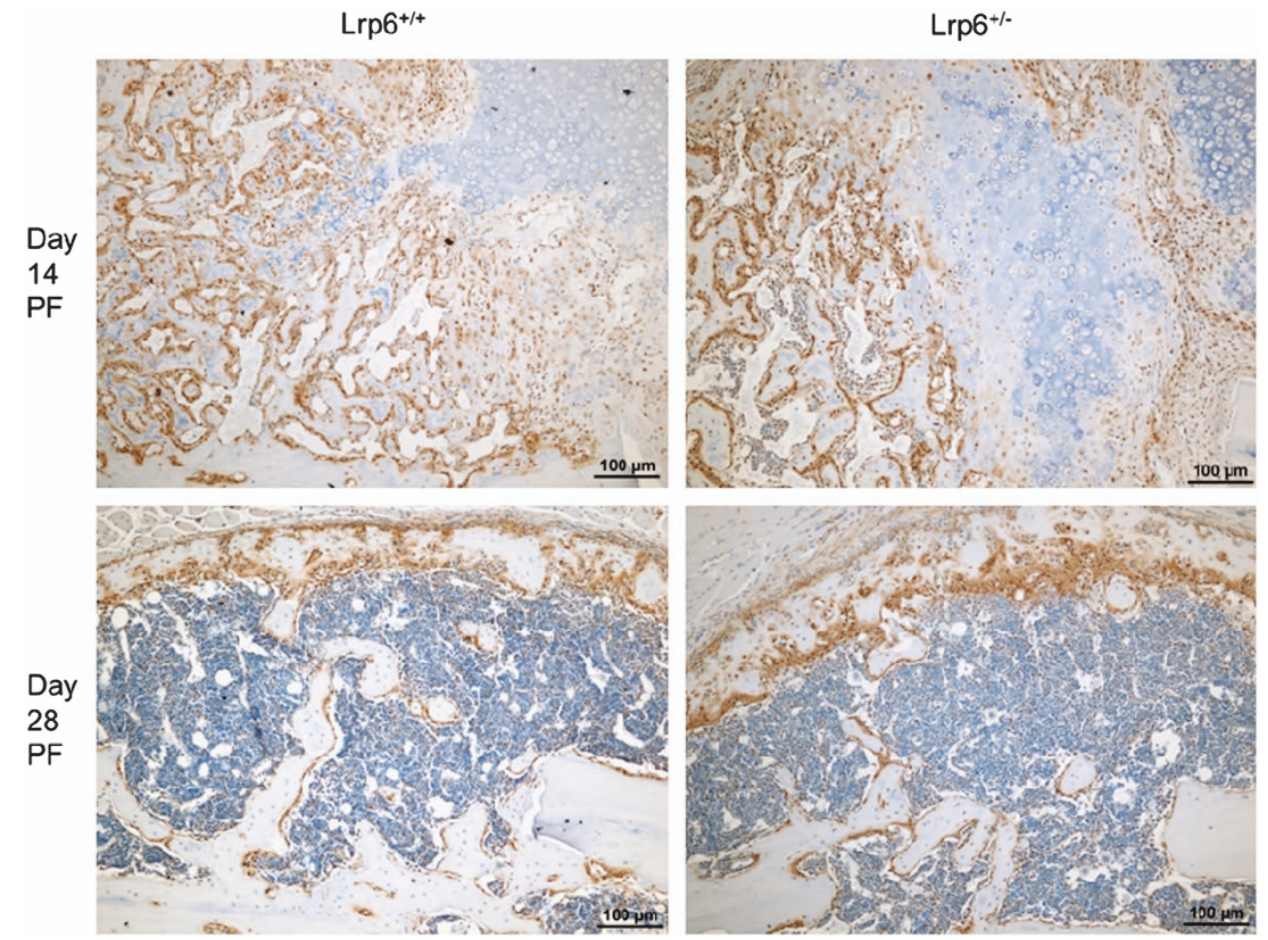

Figure 7. Representative $\beta$-catenin staining (for Wnt signaling activity, brown). There is similar $\beta$-catenin expression in the $L r p 6^{+/-}$group (left) compared with the $\mathrm{Lrp6}^{+/+}$controls (right) throughout healing (day 14 post fracture (PF), top; and day $28 \mathrm{PF}$, bottom, shown here).
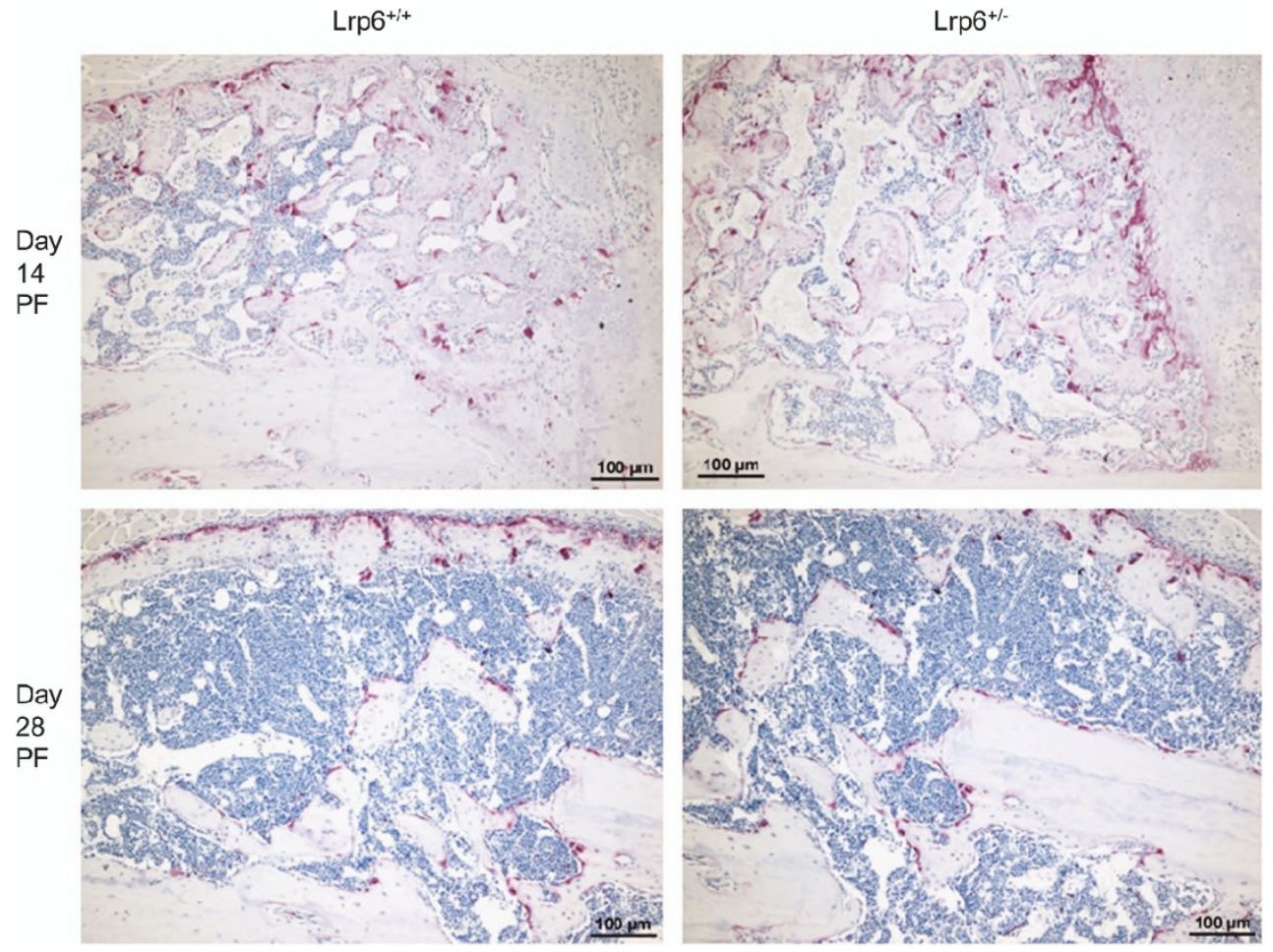

Figure 8. Representative tartrate-resistant acid phosphatase (TRAP) staining (for osteoclasts, purple). There is similar TRAP expression in the $\mathrm{Lrp6}^{+/-}$group (left) compared with the $\mathrm{Lrp6}^{+/+}$controls (right) throughout healing (day 14 post fracture (PF), top; and day $28 \mathrm{PF}$, bottom, shown here). 
$\mathrm{BV} / \mathrm{TV}, \mathrm{IV} / \mathrm{TV}$, callus density, immature density (only day 14 $\mathrm{PF})$, and mature area compared with the $\mathrm{Lrp6}^{+/+}$group; they also had significantly more empty area. By day $28 \mathrm{PF}$, there was no significant difference in any of the $\mu \mathrm{CT}$ analyses. The mature bone density decreased over time because of the change of the structure of the callus. Osteoclast activity along the original cortical bone is intense around day $21 \mathrm{PF}^{36}$ As healing progresses, the interior of the callus also loses its density as the mature bone concentrates on the periosteal surface. ${ }^{35}$ The empty (not mineralized) area in the callus was approximately doubled in the $\mathrm{Lrp6}^{+/-}$group at day $14 \mathrm{PF}$. This increase in the empty area decreased the total callus density and BV/TV in the $\mathrm{Lrp6}^{+/-}$group. The empty areas that are not mineralized are not completely empty as Figure 7 shows that the callus contains numerous cells in the regions that are not mineralized.

The mature density was likely not different between the groups because there is little gradation in the tissue density of mature bone..$^{35}$ By day $14 \mathrm{PF}$, mature bone is distributed around the outer portions of the callus, ${ }^{36}$ which is the most biomechanically advantageous location. ${ }^{8,37}$ In intact bone it is common that increased bone density leads to increased stiffness and strength, but this is not necessarily the case for fracture healing. It is most advantageous mechanically for the healing bone to be mineralized farther away from the longitudinal centerline of the bone. ${ }^{35,37}$ For example, during the healing process mineralized callus close to the original cortical bone may be mineralized, but at this location it adds little strength in a bending test. As a result, the $\mu \mathrm{CT}$ measures are useful in measuring some of the mineralization processes of the callus but should be accompanied by mechanical testing because they do not always correlate with stiffness and strength. Because there was no significant difference in the stiffness and maximum load in the callus at days 14 and $21 \mathrm{PF}$, the decrease in mature area suggests that the mature bone may have been added preferentially toward the inside of the callus.

Although the immature area was not different between the groups, the immature density was decreased at day 14 $\mathrm{PF}$ and the IV/TV was decreased at days 14 and $21 \mathrm{PF}$ in the $\mathrm{Lrpb}^{+/-}$group. This suggests that there was some mineralization delay in the immature bone in mineralizing at day $14 \mathrm{PF}$ in the $\mathrm{Lrp6}^{+/-}$group. This is consistent with a recent study reporting that Lrp6 affects early osteogenic differentiation. ${ }^{38}$ The area and density of the callus was not different between the two groups in the late stages of healing (day $28 \mathrm{PF}$ ), but the $\mathrm{Lrp6}^{+/-}$group had significantly decreased stiffness, strength, and maximum load ratio. This, along with the mineralization differences at days 14 and 21 $\mathrm{PF}$, also may indicate that the callus mineralization was more localized toward the inside of the callus where it is not as biomechanically advantageous. To enhance the understanding of the effect of Lrp6 on fracture healing, future work using finite element analysis could be used to investigate the distribution of mineral within the callus, ${ }^{39-41}$ and nano-indentation studies could be used to investigate tissue-level or location-specific differences within the callus. ${ }^{42-45}$

In a mouse model with a homozygous point mutation in Lrp6 that results in decreased bone mass, lower bone mass in the Lrp6 mutant mice was because of increased bone resorption from increased osteoclast activity with no change in bone formation. ${ }^{46}$ In an osteoblast-specific Lrp6 knockout mouse model, lower bone mass was because of decreased bone formation with no change in osteoclast activity. ${ }^{38,47}$ In this study, the qualitative appearance of the callus did not appear different between the $\mathrm{Lrp6}^{+/-}$and $\mathrm{Lrpb}^{+/+}$groups in the $\mathrm{IHC}$ staining. The progression of healing in the cartilaginous callus followed the same pattern as that of bone built during normal endochondral ossification. The qualitative similarities in $\beta$-catenin and TRAP staining indicated that there was no marked difference in Wnt signaling activity or osteoclast activity, respectively. It is possible that any difference in biological activity that may have occurred was muted because of only a single deleted allele of Lrp6.

The mechanical testing and $\mu \mathrm{CT}$ analyses from this study demonstrate the importance of Lrp6 in fracture healing and support the results of previous work on the importance of Wnt signaling in fracture healing. As embryonic mice with a homozygous Lrp6 mutation have severe skeletal deformities, ${ }^{16}$ it is likely that an Lrp6 homozygous deletion would amplify the fracture healing impairment compared with that presented in this study. It is not possible to investigate fracture healing in a total body homozygous Lrp6 deletion because these mice are not viable; however, further investigation into the effect of a homozygous deletion could be investigated in a tissue-specific level using conditional knockout mouse models using the Cre-lox system (a bone- or cartilage-specific Cre model/ ${ }^{48}$ with an $L r p 6^{\mathrm{f} / \mathrm{f}}$ model, for example, refs $\left.18,38,47,49\right)$. These conditional knockout models could also be used to further investigate whether Lrp6 impairs fracture healing due to the chondrogenic response and/or the later osteoblastic response.

\section{Acknowledgements}

We thank the members of the Mason and Williams laboratories at VARI for their assistance; Christiane Kruppa and Martin Hoffmann for assistance in performing surgeries and fractures; David Nadziejka for assistance in preparing the manuscript; Lisa Turner and the VARI histology core for their histology work; VARI's vivarium staff for outstanding animal husbandry; and Martin Alvarado, Luci Korpi, and the Grand Rapids Area Pre-College Engineering Program for helping with this research. BOW is supported by NIH grant AR053293. 


\section{Competing interests}

BOW has received honoraria for presenting seminars related to Wnt signaling at Amgen and Vertex Pharmaceuticals. The remaining authors declare no conflict of interest.

\section{References}

1 Zhong Z, Ethen NJ, Williams BO. WNT signaling in bone development and homeostasis. Wiley Interdiscip Rev Dev Biol 2014; 3: 489-500.

2 Maupin KA, Droscha CJ, Williams BO. A comprehensive overview of skeletal phenotypes associated with alterations in Wnt/beta-catenin signaling in humans and mice. Bone Res 2013; 1: 27-71.

3 Gong Y, Slee RB, Fukai N et al. LDL receptor-related protein 5 (LRP5) affects bone accrual and eye development. Cell 2001; 107: 513-523.

4 Boyden LM, Mao J, Belsky J et al. High bone density due to a mutation in LDL-receptor-related protein 5. N Engl J Med 2002; 346: 1513-1521.

5 Little RD, Carulli JP, Del Mastro RG et al. A mutation in the LDL receptor-related protein 5 gene results in the autosomal dominant high-bone-mass trait. Am J Hum Genet 2002; 70: 11-19.

6 Joiner DM, Ke J, Zhong Z et al. LRP5 and LRP6 in development and disease. Trends Endocrinol Metab 2013; 24: 31-39.

7 Azzolin L, Zanconato F, Bresolin S et al. Role of TAZ as mediator of Wnt signaling. Cell 2012; 151: 1443-1456.

8 Burgers TA, Williams BO. Regulation of Wnt/beta-catenin signaling within and from osteocytes. Bone 2013; 54: 244-249.

9 van Meurs JB, Rivadeneira F, Jhamai M et al. Common genetic variation of the low-density lipoprotein receptor-related protein 5 and 6 genes determines fracture risk in elderly white men. J Bone Miner Res 2006; 21: 141-150.

10 Mani A, Radhakrishnan J, Wang H et al. LRP6 mutation in a family with early coronary disease and metabolic risk factors. Science 2007; 315: 1278-1282.

11 Sims AM, Shephard N, Carter K et al. Genetic analyses in a sample of individuals with high or low BMD shows association with multiple Wnt pathway genes. J Bone Miner Res 2008; 23: 499-506.

12 Riancho JA, Olmos JM, Pineda B et al. Wnt receptors, bone mass, and fractures: gene-wide association analysis of LRP5 and LRP6 polymorphisms with replication. Eur J Endocrinol 2011; 164: 123-131.

13 van Meurs JB, Trikalinos TA, Ralston SH et al. Large-scale analysis of association between LRP5 and LRP6 variants and osteoporosis. JAMA 2008; 299: 1277-1290.

14 Mencej-Bedrac S, Prezelj J, Kocjan T et al. Analysis of association of LRP5, LRP6, SOST, DKK1, and CTNNB1 genes with bone mineral density in a Slovenian population. Calcif Tissue Int 2009; 85: 501-506.

15 Yerges LM, Klei L, Cauley JA et al. Candidate gene analysis of femoral neck trabecular and cortical volumetric bone mineral density in older men. J Bone Miner Res 2010; 25: 330-338.

16 Pinson KI, Brennan J, Monkley S et al. An LDL-receptor-related protein mediates Wnt signalling in mice. Nature 2000; 407: 535-538.

17 Holmen SL, Giambernardi TA, Zylstra CR et al. Decreased BMD and limb deformities in mice carrying mutations in both Lrp5 and Lrp6. J Bone Miner Res 2004; 19: 2033-2040.

18 Joeng KS, Schumacher CA, Zylstra-Diegel CR et al. Lrp5 and Lrp6 redundantly control skeletal development in the mouse embryo. Dev Biol 2011; 359: 222-229.

19 Ferguson CM, Miclau T, Hu D et al. Common molecular pathways in skeletal morphogenesis and repair. Ann N Y Acad Sci 1998; 857: 33-42.

20 Hadjiargyrou M, Lombardo F, Zhao $\mathrm{S}$ et al. Transcriptional profiling of bone regeneration. Insight into the molecular complexity of wound repair. J Biol Chem 2002; 277: 30177-30182.
21 Parker MJ, Raghavan R, Gurusamy K. Incidence of fracture-healing complications after femoral neck fractures. Clin Orthop Relat Res 2007; 458: 175-179.

22 Court-Brown CM, McQueen MM. Nonunions of the proximal humerus: their prevalence and functional outcome. J Trauma 2008; 64: 1517-1521.

23 Coulibaly MO, Sietsema DL, Burgers TA et al. Recent advances in the use of serological bone formation markers to monitor callus development and fracture healing. Crit Rev Eukaryot Gene Expr 2010; 20: 105-127.

24 French DM, Kaul RJ, D'Souza AL et al. WISP-1 is an osteoblastic regulator expressed during skeletal development and fracture repair. Am J Pathol 2004; 165: 855-867.

25 Zhong N, Gersch RP, Hadjiargyrou M. Wnt signaling activation during bone regeneration and the role of Dishevelled in chondrocyte proliferation and differentiation. Bone 2006; 39: 5-16.

26 Chen $\mathrm{Y}$, Whetstone $\mathrm{HC}$, Lin AC et al. Beta-catenin signaling plays a disparate role in different phases of fracture repair: implications for therapy to improve bone healing. PLoS Med 2007; 4: e249.

27 Komatsu DE, Mary MN, Schroeder RJ et al. Modulation of Wnt signaling influences fracture repair. J Orthop Res 2010; 28: 928-936.

28 Gaur T, Wixted JJ, Hussain S et al. Secreted frizzled related protein 1 is a target to improve fracture healing. J Cell Physiol 2009; 220: 174-181.

29 Kelly OG, Pinson KI, Skarnes WC. The Wnt co-receptors Lrp5 and Lrp6 are essential for gastrulation in mice. Development 2004; 131: 2803-2815.

30 Bonnarens F, Einhorn TA. Production of a standard closed fracture in laboratory animal bone. J Orthop Res 1984; 2: 97-101.

31 Hugunin KM, Fry C, Shuster K et al. Effects of tramadol and buprenorphine on select immunologic factors in a cecal ligation and puncture model. Shock 2010; 34: 250-260.

32 Burgers TA, Hoffmann MF, Collins CJ et al. Mice lacking pten in osteoblasts have improved intramembranous and late endochondral fracture healing. PLoS One 2013; 8: e63857.

33 Rho JY, Hobatho MC, Ashman RB. Relations of mechanical properties to density and CT numbers in human bone. Med Eng Phys 1995; 17: 347-355.

34 Burgers TA, Mason J, Niebur G et al. Compressive properties of trabecular bone in the distal femur. J Biomech 2008; 41: 1077-1085.

35 Collins CJ, Vivanco JF, Sokn SA et al. Fracture healing in mice lacking Pten in osteoblasts: a micro-computed tomography image-based analysis of the mechanical properties of the femur. J Biomech 2015; 48: 310-317.

36 Ushiku C, Adams DJ, Jiang X et al. Long bone fracture repair in mice harboring GFP reporters for cells within the osteoblastic lineage. J Orthop Res 2010; 28: 1338-1347.

37 Aiyangar AK, Au AG, Crenshaw TD et al. Recovery of bone strength in young pigs from an induced short-term dietary calcium deficit followed by a calcium replete diet. Med Eng Phys 2010; 32: 1116-1123.

38 Riddle RC, Diegel CR, Leslie JM et al. Lrp5 and Lrp6 exert overlapping functions in osteoblasts during postnatal bone acquisition. PLoS One 2013; 8: e63323.

39 Gardner TN, Mishra S. The biomechanical environment of a bone fracture and its influence upon the morphology of healing. Med Eng Phys 2003; 25: 455-464.

40 Vaughan TJ, McCarthy CT, McNamara LM. A three-scale finite element investigation into the effects of tissue mineralisation and lamellar organisation in human cortical and trabecular bone. J Mech Behav Biomed Mater 2012; 12: 50-62.

41 Trabelsi N, Milgrom C, Yosibash Z. Patient-specific FE analyses of metatarsal bones with inhomogeneous isotropic material properties. J Mech Behav Biomed Mater 2014; 29: 177-189.

42 Leong PL, Morgan EF. Correlations between indentation modulus and mineral density in bone-fracture calluses. Integr Comp Biol 2009; 49: 59-68. 
43 Spiesz EM, Roschger P, Zysset PK. Elastic anisotropy of uniaxial mineralized collagen fibers measured using two-directional indentation. Effects of hydration state and indentation depth. J Mech Behav Biomed Mater 2012; 12: 20-28.

44 Rodriguez-Florez N, Oyen ML, Shefelbine SJ. Insight into differences in nanoindentation properties of bone. J Mech Behav Biomed Mater 2013; 18: 90-99.

45 Slane J, Vivanco J, Ebenstein D et al. Multiscale characterization of acrylic bone cement modified with functionalized mesoporous silica nanoparticles. J Mech Behav Biomed Mater 2014; 37: 141-152.

46 Kubota T, Michigami T, Sakaguchi N et al. Lrp6 hypomorphic mutation affects bone mass through bone resorption in mice and impairs interaction with Mesd. J Bone Miner Res 2008; 23: 1661-1671.

47 Li C, Xing Q, Yu B et al. Disruption of LRP6 in osteoblasts blunts the bone anabolic activity of PTH. J Bone Miner Res 2013; 28: 2094-2108.
48 Elefteriou F, Yang X. Genetic mouse models for bone studies--strengths and limitations. Bone 2011; 49: 1242-1254.

49 Li C, Williams BO, Cao X et al. LRP6 in mesenchymal stem cells is required for bone formation during bone growth and bone remodeling. Bone Res 2014; 2: 14006.

\section{(c) (i)}

This work is licensed under a Creative Commons Attribution 4.0 International License. The images or other third party material in this article are included in the article's Creative Commons license, unless indicated otherwise in the credit line; if the material is not included under the Creative Commons license, users will need to obtain permission from the license holder to reproduce the material. To view a copy of this license, visit http://creativecommons.org/licenses/by/4.0/

(C) The Author(s) 2016 